\title{
Challenges in diabetic foot treatment during pandemic of COVID-19
}

Haris Vukas, MD, MoS, Dragan Piljic, MD, PhD, Samra Kadić-Vukas, MD, Dilista Piljic, MD, PhD.

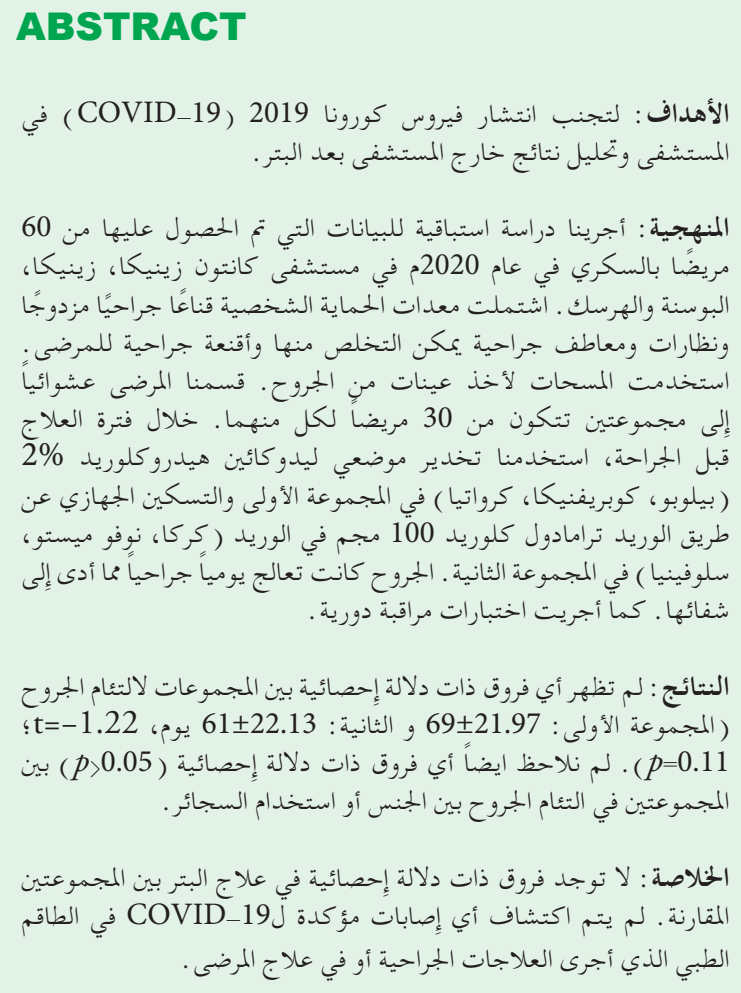

Objectives: To avoid hospital spread of Coronavirus-2019 (COVID-19) and to analyze out of hospital outcomes after amputation.

Methods: Prospective analysis of data obtained from 60 diabetic patients in 2020 was performed at Cantonal Hospital Zenica, Zenica, Bosnia and Herzegovina. Personal protection equipment included double surgical mask, glasses, disposable surgical coats, and surgical masks for patients. Swabs were used to take samples from wounds. We randomly divided patients in 2 groups of 30 patients each. In preoperative treatment, we used local anesthesia lidocaine hydrochloride 2\% (Belupo, Koprivnica, Croatia) in group A and systemic analgesia intravenous tramadol chloride $100 \mathrm{mg}$ intravenous (Krka, Novo Mesto, Slovenia) in group B. Wounds were surgically treated each day and heal spontaneously. Periodical control exams were performed.

Results: Wound healing did not present any statistically significant differences between groups (group A: $69 \pm 21.97$ and $\mathrm{B}$ : $61 \pm 22.13$ days, $\mathrm{t}=-1.22$; $p=0.11)$. No statistically significant differences $(p<0.05)$ between groups A and B in wound healing regarding to gender or cigarette use was noted.

Conclusion: No significant differences in amputation treatment between the 2 comparative groups were noted. No confirmed COVID-19 infections in medical staff who performed surgical interventions or in treated patients were detected.

Keywords: diabetic foot, amputation, COVID-19

Saudi Med J 2021; Vol. 42 (2): 166-169 doi: 10.15537/smj.2021.2.25687

From the Department of Vascular Surgery (Vukas), from the Department of Neurology (Kadić-Vukas), Cantonal Hospital Zenica, Zenica; from the Department of Vascular Surgery (Dragan Piljic), Cardiovascular Surgery Clinic, and from the Intensive Care (Dilista Piljic), University Clinical Center Tuzla, Clinic of Infective Disease, Tuzla, Bosnia and Herzegovina.

Received 17th November 2020. Accepted 30th December 2020.

Address correspondence and reprint request to: Dr. Haris Vukas, Department of Vascular Surgery, Cantonal Hospital Zenica, Zenica, Bosnia and Herzegovina.Email: haris.vukas@ssst.edu.ba ORCID ID: https://orcid.org/0000-0001-5061-6661 
$\mathrm{O}$ ur hospital is small with limited resources, and the total number of anesthesiologist specialists is 12. Until now, 7 anesthesiologists were infected with Corononavirus-2019 (COVID-19) and presented with severe clinical condition. For our hospital, every day is a challenge to provide sufficient medical care to patients. Due to insufficient testing resources, we have lesser possibility of confirming the COVID-19 infection in patients from Canton Zenica-Doboj (area of $3.343 .3 \mathrm{~km}^{2}$, population 360.093 [119 citizens/ $\left.\mathrm{km}^{2}\right]$ ).

Morbidity due to diabetes mellitus in 2016 was $336.5 / 10,000$, and in 2017 , it was $324.3 / 10,000$ citizens. Among the 5 leading diseases in 2017, hypertensive diseases had $13 \%$ of the total registered diseases with a disease rate of 1.217/10,000 inhabitants, and diabetes mellitus had a share of $3.7 \%$ with a disease rate of 349/10,000 inhabitants.

The fifth most common cause of death in women in 2017 was insulin-independent diabetes mellitus at a rate of 51.8 per 100,000 citizens in the Federation of Bosnia and Herzegovina. ${ }^{1}$ In pandemic of COVID-19, we are facing an increase rates of serious clinical presentations and complications of diabetic mellitus. The lockdown has impacted on low physical activity, lesser contact with medical staff, and increased stress factors which have direct negative impact on patients health. Now we are facing with enormous problems of how to provide sufficient health care and treatment to diabetic patients. Diabetic foot is a severe public health issue worldwide with direct implications on healthcare budgets. Diabetic foot ulcer prevalence was 6.3\% (95\% confidence interval [CI]: 5.4-7.3\%), which was higher in males $(4.5 \%$, 95\%CI: $3.7-5.2 \%)$ than in females $(3.5 \%, 95 \% \mathrm{CI}$ : $2.8-4.2 \%)$, and higher in type 2 diabetic patients $(6.4 \%$, 95\%CI: $4.6-8.1 \%)$ than in type 1 diabetics $(5.5 \%$, 95\%CI: 3.2-7.7\%). North America had the highest prevalence of diabetic foot $(13.0 \%, 95 \% \mathrm{CI}$ : 10.0 $15.9 \%){ }^{2}$ Infection, ulceration, or destruction of tissues was found in $6 \%$ of diabetic patients $(0.03 \%-1.5 \%$ of patients require an amputation). Diabetic foot signs are diverse and include perforating strand, various forms of fasciitis, abscesses, osteitis, cellulitis, and both dry and wet (moist) gangrene. ${ }^{3,4}$

Cantonal Hospital Zenica is faced with the high-risk situation of admitting diabetic foot patients into the

Disclosure. Authors have no conflict of interests, and the work was not supported or funded by any drug company. hospital without resources for Coronavirus-2019 testing (COVID-19) using polymerase chain reaction (PCR). Our goal was to avoid hospital spread of COVID-19 and to analyze out of hospital outcomes after amputation.

Methods. The study was prospective, descriptiveanalytical, comparative, randomized, and conducted in the vascular laboratory ambulance of Cantonal Hospital Zenica, Zenica, Bosnia and Herzegovina between January 2020 and September 2020. The ethical approval was obtained from the Ethical Committee of Cantonal Hospital Zenica. The inclusion criteria for patients were: diabetes mellitus, foot finger gangrene (wet or dry) with or without phlegmona and neurotrophic wounds. Exclusion criteria were: non diabetic patients, patients with malignant disease, foot traumatic injuries and burns.

All procedures performed in the studies involving human participants were in accordance with the ethical standards of the institutional or national research committee and with the 1964 Helsinki Declaration and its later amendments or comparable ethical standards.

Patients were randomly divided into 2 groups each with 30 patients. In the pre-operative treatment we used local anesthesia lidocaine hydrochloride $2 \%$ sub cutaneously (200-400 mg) (Belupo, Koprivnica, Croatia) in group A and systemic analgesia, intravenous tramadol chloride $100 \mathrm{mg}$ intravenous (Krka, Novo Mesto, Slovenia) in group B.

Swabs were used to take bacterial samples from wounds. Blood flow was confirmed, with color Doppler (Philips, Amsterdam, Netherlands) through femoral, popliteal, and pedal arteries. X-rays (Philips, Amsterdam, Netherlands) of the feet were performed in non-febrile cases that had also negative epidemiological survey. Patients had gangrene, some with one finger $(n=50)$, 2 fingers $(n=8)$ and 3 fingers $(n=2)$. We identify foot phlegmona in 40 patients. Septic signs were present in 37 patients and 3 patients had severe septic condition. Wounds were treated daily with $10 \%$ povidone iodine (Bosnalijek, Sarajevo, Bosnia and Herzegovina) and sulfadiazine silver $10 \mathrm{mg} / \mathrm{g}$ (Bosnalijek, Sarajevo, Bosnia and Herzegovina) and surgical wound debridement was conducted by house care medical teams. Enteral systemic antibiotics, Amoxicillin 2 grams/per day, Ciprofloxacin $1000 \mathrm{mg} /$ day, Metronidazole $1000 \mathrm{mg} /$ day for 7 to 14 days (Bosnalijek, Sarajevo, Bosnia and Herzegovina), were administered only in cases of local or systemic septic signs. With intention, wounds were allowed to heal spontaneously. Control exams were done every 21 days, and patients were followed for 3 to 4 months. 
During amputation, all patients had surgical masks that covered their mouths and noses. Patient heads were separated from the surgeon with a cotton cloth. All medical staff had surgical masks, visors, and disposable surgical uniforms. After interventions, we used chlorine solution and ultraviolet (UV) lamps for disinfection.

The polymerase chain reaction (PCR) test for COVID-19 in patients was not performed.

Statistical analysis. All results were analyzed by the statistical program SPSS version 16 (SPSS Inc. Released 2007. SPSS for Windows, Version 16.0. Chicago, SPSS Inc). The results were analyzed using t-test and HI2 test for comparison between the investigated groups. The degree of correlation was tested using the correlation coefficient Pearson or Spearman. Finally, it would apply the appropriate models of regression analysis to determine the independent association of variables. Values of $p<0.05$ will be considered statistically significant.

Results. No statistically significant differences in age between groups (standard deviation: group $A=11.74$,

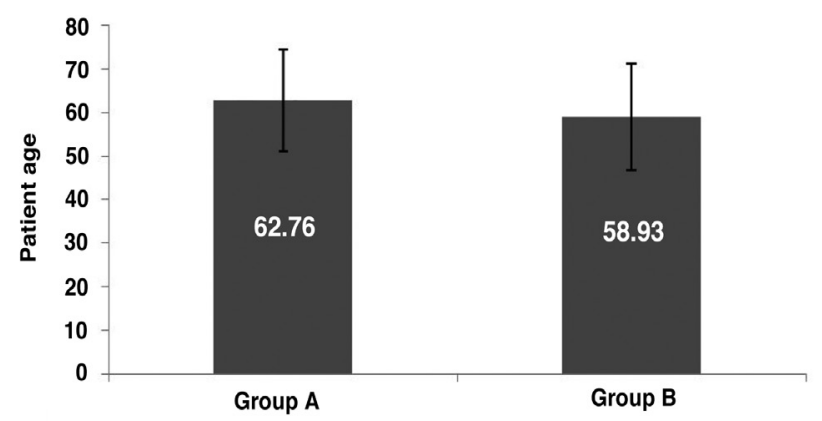

Figure 1 - Age of patients.

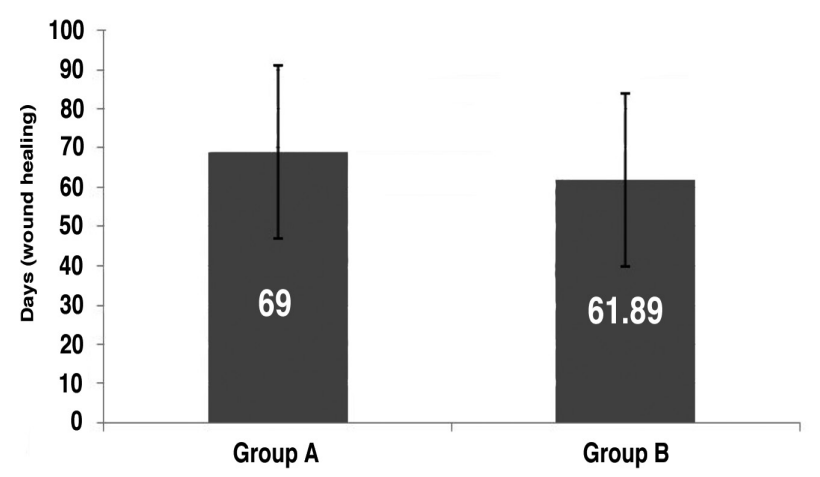

Figure 2 - Wound healing in days
Table 1 - Patient genders, risks, therapies, and local findings.

\begin{tabular}{lccc}
\hline Variables & Group A & Group B & Total \\
\hline Female & $21(63.3)$ & $19(70.0)$ & $40(66.7)$ \\
Cigarette smoking & $13(36.7)$ & $18(60.0)$ & $31(51.7)$ \\
Insulin & $23(76.7)$ & $21(70.0)$ & $44(73.3)$ \\
ACE inhibitors & $24(80.0)$ & $27(90.0)$ & $51(85.0)$ \\
Statins & $23(76.7)$ & $29(96.7)$ & $52(86.7)$ \\
Antithrombotics & $27(90.0)$ & $30(100)$ & $57(95.0)$ \\
Antibiotics & $26(86.7)$ & $26(86.7)$ & $52(85.0)$ \\
Dry gangrene & $8(26.7)$ & $7(23.3)$ & $15(25.0)$ \\
Wet gangrene & $22(73.3)$ & $23(76.7)$ & $45(75.0)$ \\
First amputation & $18(60.0)$ & $20(66.7)$ & $38(63.3)$ \\
$\begin{array}{l}\text { Fingers } \\
\text { re-amputation }\end{array}$ & $8(26.7)$ & $1(3.3)$ & $9(15.0)$ \\
\hline
\end{tabular}

Values are presented as numbers and percentages (\%). No significant difference between groups with respect to gender, risks, therapies, or local findings were noted.

group $\mathrm{B}=12.25$ ) were noted as shown in Figure 1. Wound healing tendencies did not show statistical significance difference between groups (standard deviation [SD]: group A 21.97, group B 22.13, $\mathrm{t}=-1.22 ; p=0.11$, Figure 2). Two patients, one in each group, completed treatment with amputations above knee.

No statistical differences in glycosylated hemoglobin (HbAlc) findings between group $\mathrm{A}$ and $\mathrm{B}$ : mean 8.83 versus 8.89 ( $\mathrm{SD}$ : group $\mathrm{A}=2.01$, group $\mathrm{B}=1.56$ ), standard error 0.37 versus [vs.] $0.29(p<0.05)$. Four patients in group $A$ and 3 patients in group B did not have $\mathrm{HbA1c}$ measurements. No statistically significant difference $(p<0.05)$ between groups A and B in wound healing with respect to gender $(\mathrm{t}=-1.09, p=0.15$ vs. $\mathrm{t}=-0.27, p=0.39)$, cigarette use $(\mathrm{t}=-1.05, p=0.15$ vs. $\mathrm{t}=-0.73, p=0.23)$, first amputation $(\mathrm{t}=0.39, p=0.35$ vs. $\mathrm{t}=-1.32, p=0.09)$, dry gangrene $(\mathrm{t}=-0.25, p=0.39$ vs. $\mathrm{t}=-0.93, p=0.18)$, wet gangrene $(\mathrm{t}=-0.93, p=0.18$ vs. $\mathrm{t}=0.26, p=0.40)$, antibiotics $(\mathrm{t}=1.17, p=0.12$ vs. $\mathrm{t}=0.39, p=0.34)$, insulin therapy $(\mathrm{t}=-0.87, p=0.2$ vs. $\mathrm{t}=-0.9, p=0.04)$, antibiotic therapy $(\mathrm{t}=-0.64, p=0.27$ vs. $\mathrm{t}=-1.27, p=0.1)$.

Discussion. We confirmed in our study that the main variables were postoperative wound care (depended on personal professionalism of medical nurses), social, and living conditions. We did not measure the possible pain that the patient felt. In addition, toxicity of local anesthetics and their influence on increasing unwanted 
outcomes after foot finger amputations compared to the comparative group were not confirmed.

Diabetic nerves are more sensitive to local anesthetics, toxicity, and increased possibility of infection using nerve catheter. ${ }^{5}$ In general, doses and concentrations of local anesthetics administered in clinical practice today are lower than before; nonetheless, Yu et $\mathrm{al}^{6}$ confirmed that although small, risk of local anesthetic toxicity, even in healthy nerves, exists.

We still need to be aware of side effects and complications of local anesthetics, such was found in a 63-year-old female with history of smoking abuse, who after an injection of lidocaine with epinephrine, developed necrosis distal phalanx of 2 fingers that required amputations. ${ }^{7}$ Regardless our study results, we suggested that using local anesthetics in preoperative preparation for finger amputation is safer and desirable, although we cannot confirm antimicrobial effects of $2 \%$ lidocaine hydrochloride and its role on better healing process.

In conclusion, our research goal was due to the COVID-19 pandemic to explore amputation outcomes performed with limited resources. We also observe risk and possibility of COVID-19 contamination and infection of medical staff and patients. We observed and found that after use of local anesthetics versus intravenous analgesia there were no significant differences between groups in healing outcomes. Healthcare services that we provide are far from ideal and sufficient, but we need to be aware that we must provide medical service to help diabetic patients in every way possible considering our resources. No confirmed cases of COVID-19 infections on medical staff who performed surgical interventions or patients who underwent treatment.

Acknowledgment. We would like to thank American Manuscript Editors (https:/lamericanmanuscripteditors.com) for English language editing.

\section{References}

1. Institute for Public Health FB\&H. Health condition of the population and health care in the Federation of the Bosnia and Herzegovina. [Updated: 2017. Accessed 2021 January 20] Available from URL: https://www.zzjzfbih.ba/wp-content/ uploads/2018/10/Zdravstveno-2017.pdf

2. Zhang P, Lu J, Jing Y, Tang S, Zhu D, Bi Y. Global epidemiology of diabetic foot ulceration: a systematic review and meta-analysis. Ann Med 2017; 49: 106-116.

3. Mishra SC, Chhatbar KC, Kashikar A, Mehndiratta A. Diabetic foot. BMJ 2017; 359: j5064.

4. Ţânţu MM, Man GM, Rogozea LM, Domnariu CD, Pleşa FC, Traşcă DM, et al. Diabetic foot-epidemiological and histopathological aspects. Rom J Morphol Embryol 2018; 59: 895-902.

5. Ten Hoope W, Looije M, Lirk P. Regional anesthesia in diabetic peripheral neuropathy. Curr Opin Anaesthesiol 2017; 30: 627-631.

6. Yu ZY, Geng J, Li ZQ, Sun YB, Wang SL, Masters J, et al. Dexmedetomidine enhances ropivacaine-induced sciatic nerve injury in diabetic rats. Br J Anaesth 2019; 122: 141-149.

7. Zhang JX, Gray J, Lalonde DH, Carr N. Digital necrosis after lidocaine and epinephrine injection in the flexor tendon sheath without phentolamine rescue. J Hand Surg Am 2017; 42: e119-e123. 